\title{
EFFECTS OF DIFFERENT TYPES OF INPUT WAVEFORMS IN PATIENT-SPECIFIC RIGHT CORONARY ATHEROSCLEROSIS HEMODYNAMICS ANALYSIS
}

\author{
S.I. BERNAD ${ }^{1}$, E.S. BERNAD ${ }^{2}$, T. BARBAT ${ }^{1}$, V. ALBULESCU ${ }^{2}$ \& R. SUSAN-RESIGA ${ }^{3}$ \\ ${ }^{1}$ Romanian Academy - Timisoara Branch, Romania. \\ ${ }^{2}$ University of Medicine and Pharmacy "Victor Babes" Timisoara, Romania. \\ ${ }^{3}$ Department of Hydraulic Machinery, "Politehnica" University of Timisoara, Romania.
}

\begin{abstract}
Accurate fluid mechanics models are important tools for predicting the flow field in the coronary artery for understanding the relationship between hemodynamics and the initiation and progression of atherosclerosis. The purpose of this paper is to asses non-invasively hemodynamic parameters such as disturbed flows, pressure distribution and wall shear stress with computational fluid dynamics (CFD) in human right coronary artery (RCA) using patient-specific data from in vivo computed tomographic (CT) angiography, using two different pulsatile input waveforms. In order to produce a realistic three-dimensional model of the RCA anatomy, CT-datasets were acquired by a four-row-detector CT-scanner. Digital files in Digital Imaging and Communications in Medicine (DICOM) file format, containing cross-sectional information were then imported to CFD software package for reconstruction. The numerical analysis examines closely the effect of a different input waveforms model on the hemodynamic characteristics such as secondary flow, flow separation and wall shear stress in the multiple stenosed RCA.

Keywords: hemodynamics, input velocity, wall shear stress, stenotic arteries, blood flow distorsion, secondary flow, flow separation.
\end{abstract}

\section{INTRODUCTION}

Atherosclerosis is a disease of large and medium-size arteries, which involves complex interactions between the artery wall and the blood flow. Both clinical reports and numerical simulations show that hemodynamics plays an important role in the pathogenesis of atherosclerosis [1-5]. It is widely believed that atherosclerosis development and progression are affected by many risk factors, such as static pressure, wall shear stress (WSS), blood viscosity, flow velocity, etc. Vascular geometry is another factor influencing strongly the flow patterns and generating a pre-existing atherogenic hemodynamic environment [6-9].

It is now well known that human atherosclerotic lesions originate preferably at the outer wall of vessel branches or bifurcations and at the inner wall of artery bends, where the blood vessel curvature creates dynamic environments of disturbed flow [6].

Although there are many factors that regulate the vascular remodeling, the endothelial cell and its interaction with the fluid-induced WSS play a key role in the adaptive response of vessels. The fluidinduced WSS is a very small frictional force induced by a viscous fluid flow moving on the surface of solid materials. The endothelial cell is capable of sensing the level and the direction of the WSS induced by the blood flow, and the biomechanical properties of endothelial cell are partially regulated by the WSS [2, 9-11].

Non-invasive imaging techniques coupled with CFD simulations are important tools for studying the flow field in individual human arterial models, and these numerical models are a convenient platform for evaluating the influence of vessel geometry on pathophysiologically relevant biomechanical properties $[3,5,12]$.

A detailed hemodynamic evaluation of disturbed flow in the post-stenosis region may give additional insight to understand the progression of atherosclerosis and may have useful clinical value, such as early detection of a highly stenosed artery segment, prediction of future disease progression, and treatment planning $[1,13]$. 


\section{COMPUTATIONAL FLUID DYNAMICS IN VASCULAR CARDIOLOGY}

Over the past two decades, the fluid mechanics of blood flow has been shown to play an important role in arterial adaptation and disease localization [14]. Flow patterns present within complex arterial geometries can be examined through experimental flow studies using large-scale in vitro models. These types of studies are also important for evaluation of diagnostic and therapeutic medical devices and flow measurement techniques. Furthermore, these types of studies are important for the validation of computational fluid dynamics (CFD) codes. With advances in computational speed and CFD codes, numerical simulation of blood flow has become a powerful tool.

Advances in CFD and computer technology are increasing the possibilities of simulation of blood flow in the cardiovascular system. CFD software provides a fast, non-invasive method of assessing surgical techniques, analyzing arterial diseases and examining blood flow in a number of diseased conditions [15-18]. These computational techniques can be used to predict the outcome of surgical and interventional procedures aiding the physicians to decide which is the best treatment for a given individual. Computer simulations can help to design less invasive and more precise procedures, to increase the quality and cost-effectiveness of the therapy and to provide better training for physicians.

\section{MATERIALS AND METHOD}

For the case presented in this paper, spiral CT (computed tomography) was performed for 4 days following the CA (coronary angiography) (44 year old, patient with typical angina symptoms is investigated). A Somatom Sensation 64 Scanner (Siemens Medical Systems, Erlangen, Germany) was used in non-enhanced spiral scan technique with a slice thickness of $2 \mathrm{~mm}$, a table feed of $3 \mathrm{~mm} / \mathrm{s}$, and an increment of $2 \mathrm{~mm}$. Data corresponding to the investigated patient is presented in Table 1.

The CA and the spiral CT investigation detected a multiple severe right coronary artery (RCA) stenosis (Fig. 1). According to the NASCET (North American Symptomatic Carotid Endarterectomy Trial) [19] and ECST (European Carotid Surgery Trial) [20] method of stenosis classification, the stenoses severity is illustrated in Table 2.

The percentage diameter reduction for a circular stenosis is $1-d / D$, where $d$ is the diameter of the lumen and $D$ the diameter of the unoccluded artery (for the present case diameter of section D3).

Table 1: Preoperative patient characteristics.

\begin{tabular}{ll}
\hline Variables & Value \\
\hline Mean age & 44 \\
Gender (M/F) & Male \\
History of MI & No \\
Previous PTCA & No \\
Renal insufficiency & No \\
Cardiovascular risk factor & \\
Hypertension & Yes \\
Diabetes & No \\
Smoke & Yes \\
Obesity & Moderate \\
Angiographic data & \\
RCA stenosis & Multiple/severe \\
\hline
\end{tabular}

$\mathrm{MI}$ = myocardial infarction; $\mathrm{PTCA}=$ percutaneous transluminal coronary angioplasty; $\mathrm{RCA}=$ right coronary artery. 


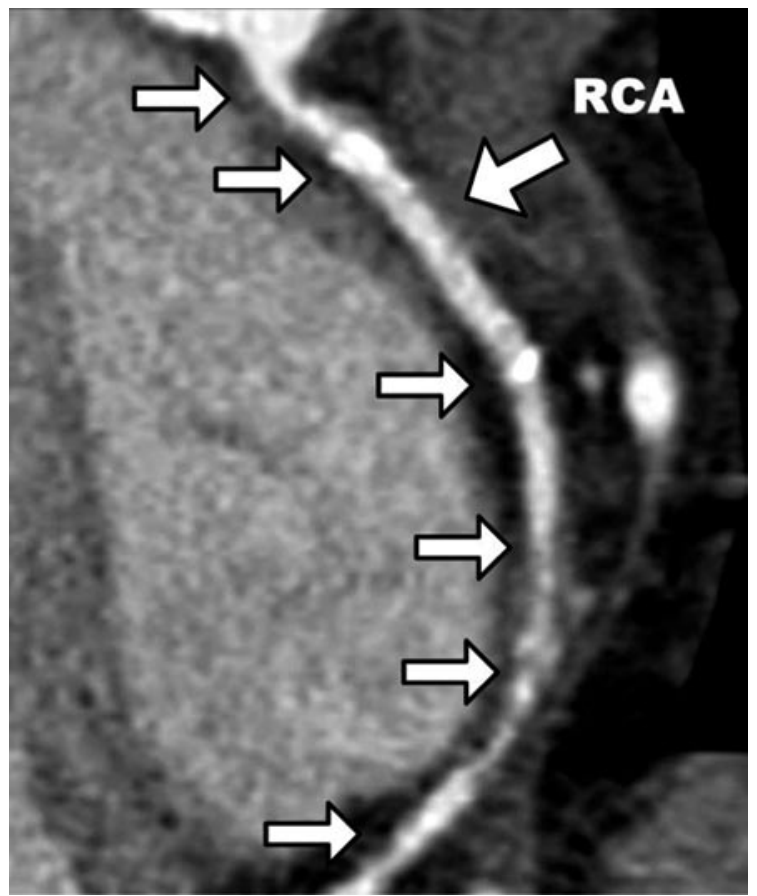

Figure 1: Patient-specific right coronary artery (RCA). Axial tomographic image which indicates a multiple coronary stenosis at the RCA (arrows) (64-slice CT).

Table 2: RCA stenosis severity (Fig. 2).

\begin{tabular}{lccc}
\hline Section & $\begin{array}{c}\text { Section diameter } \\
(\mathrm{mm})\end{array}$ & $\begin{array}{c}\text { Section area } \\
\left(\mathrm{mm}^{2}\right)\end{array}$ & $\begin{array}{c}\text { Diameter reduction } \\
\text { percentage }(\%)\end{array}$ \\
\hline D0 & 3.8 & 11.3267 & - \\
D1 & 1.76 & 2.4621 & 55 \\
D2 & 1.81 & 2.5734 & 54 \\
D3 & 3.96 & 11.7578 & - \\
D4 & 2.6 & 5.3025 & 34 \\
D5 & 3.84 & 11.3928 & - \\
D6 & 1.68 & 2.2361 & 57 \\
D7 & 3.6 & 10.1818 & - \\
D8 & 1.6 & 2.008 & 60 \\
\hline
\end{tabular}

\subsection{Computational geometry}

A computer aided design (CAD) model of a human RCA lumen was reconstructed from CT scans of a patient. The patient was a 44-year-old male who had a clinically identified stenosis in his RCA. The RCA geometry was reconstructed based on CT images. Images were then segmented in crosssections perpendicular to the RCA centerline. Figure 1 shows the reconstructed lumen geometry of the RCA viewed from the epicardial, and pericardial sides. The lumen inlet diameter (hydraulic diameter) of the RCA was $3.8 \mathrm{~mm}$. The RCA is modeled to be $52 \mathrm{~mm}$ in length with variable diameters, 


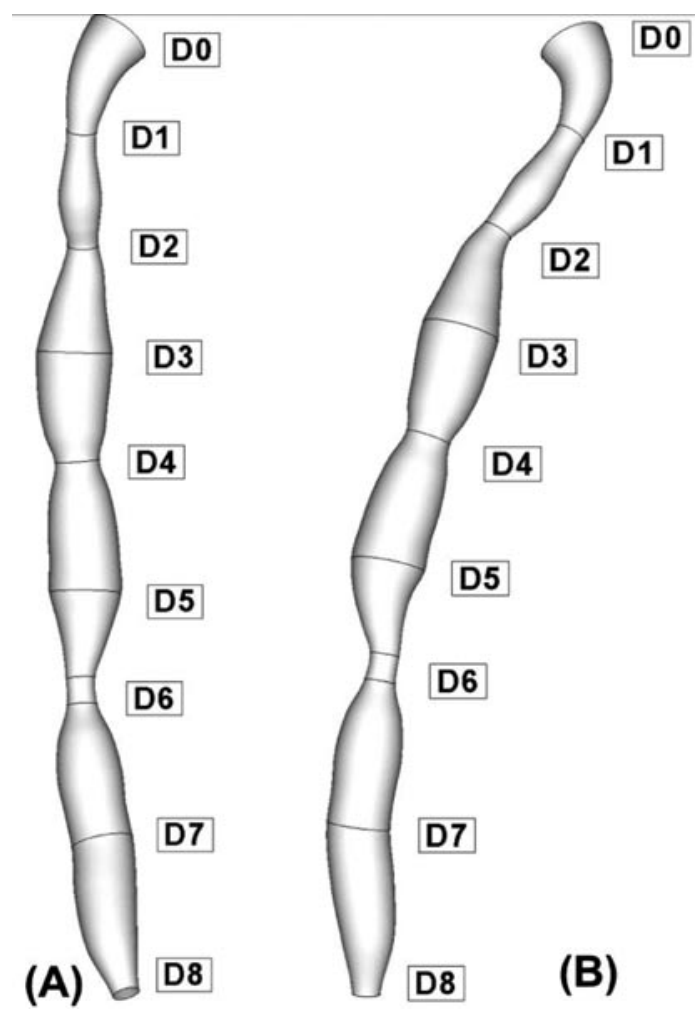

Figure 2: Patient-specific right coronary artery (RCA) geometry reconstruction. Reconstructed 3D geometry: (a) pericardial side; (b) epicardial side.

depending on the stenosis severity. Data corresponding to the reconstructed RCA is presented in Table 2. Good agreement has been demonstrated between coronary artery diameter measurements in the present case and the measurements done by transthoracic echocardiography (TTE) and quantitative coronary angiography (QCA) presented by Kiviniemi et al. [21].

An effective meshing procedure in this study is one that can mesh the CT scan image surface geometry without compromising the geometric features. To achieve this task the commercial grid generation software, GAMBIT 2.4.6 (ANSYS FLUENT, ANSYS, Inc.) was utilized [22].

The elements employed to mesh the 3D computational domain of the coronary arterial segment, consisted primarily of regular structured hexahedral elements as well as wedge elements wherever necessary. In order to carry out the mesh sensitivity analysis, numerical simulations were carried out by varying the number of mesh elements in the computational domain. The accuracy of the simulation results was then improved by employing a finer mesh that contained 1,598,752 elements. Figure 3 shows an enlarged view of the reconstructed mesh for the flow dynamic analysis.

\subsection{Model assumptions}

The blood is assumed to be incompressible, with a Newtonian behavior having dynamic viscosity $(\mu)$ of $0.00408 \mathrm{~Pa}$ and a density $(\rho)$ of $1050 \mathrm{~kg} / \mathrm{m}^{3}$. Johnston et al. [23] compared the effects of different blood viscosity models on the WSS distributions in the RCA during the cardiac cycle. Their study 


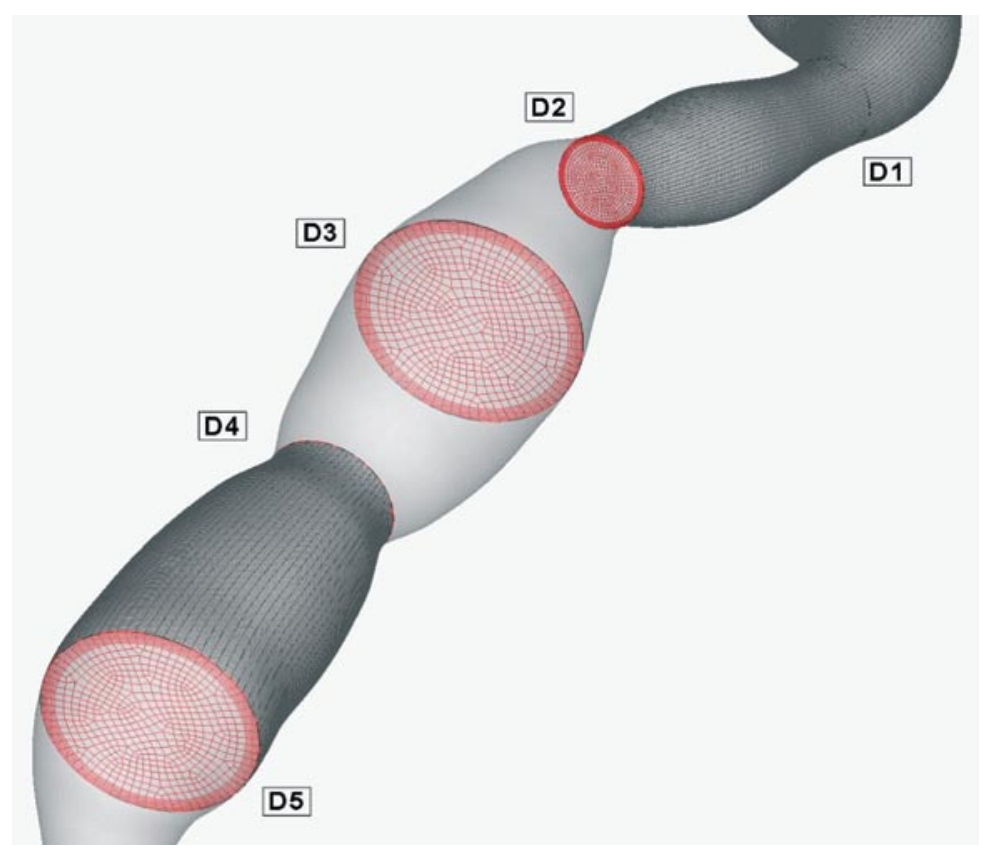

Figure 3: Reconstructed right coronary artery. Computational domain discretization using boundary layer techniques.

showed that the use of a Newtonian blood model is a reasonably good approximation when studying the WSS distribution for transient blood flow in arteries. The wall motion associated with pressure and flow pulsation is believed to be relatively small in constrictions with thickened intima because of the decreased flexibility of the wall. The walls were taken as solid and stiff, and a zero-velocity boundary condition was assumed for the walls, corresponding to a no-slip condition.

The governing equations of motion for the flow dynamic solution, i.e., the continuity equation and the time dependent 3D Navier-Stokes equations for an incompressible viscous fluid were applied in the numerical analysis. Commercial CFD FLUENT 6.3 package [22], parallelized across eight $3.2 \mathrm{GHz}$ Intel processors of a TYANPSC T-650 Rx (Tyan Computer Corporation, Taiwan, parallel computing machine with sixteen $3.2 \mathrm{GHz}$ processors) is used in order to perform the numerical analysis.

Three cycles were simulated to ensure that the flow was truly periodic [24]. Computations were repeated over different meshes to ensure that the numerical solutions were mesh independent. Computations were carried out with various values of physiological parameters to reveal the detailed characteristics of the flow in curved stenotic arteries and to examine the influence of stenosis on the blood flow pattern, including flow separation, secondary flow, WSS level and pressure drop. The time step employed for the unsteady simulations in the study is $0.001 \mathrm{~s}$ has been equivalent to using 1000 time steps to compute a single cardiac cycle.

Simulation results were visualized with TECPLOT (Tecplot, Inc., Bellevue, WA).

\subsection{Flow conditions}

In order to evaluate the effects of input function on flow patterns and WSS variation, we used two types of waveform models at the inlet section of the RCA geometry: 
Case 1: Pulsatile inlet flow, which is a more realistic approximation of physiologic waveforms [25] presented in eqn (1), since it has a higher peak-to-mean ratio, is obtained using two harmonics and with $a_{1}=-a_{2}=0.75$ (Fig. 4).

$$
\bar{u}(t)=\bar{u}_{m}\left[1+a_{1} \sin \left(\frac{2 \pi t}{T}\right)+a_{2} \cos \left(\frac{4 \pi t}{T}\right)\right]
$$

Case 2: Pulsatile inlet flow, which is the physiologically realistic case, based on flow and pressure waveforms acquired with an intravascular ultrasound Doppler probe in the RCA [26] (Fig. 5).

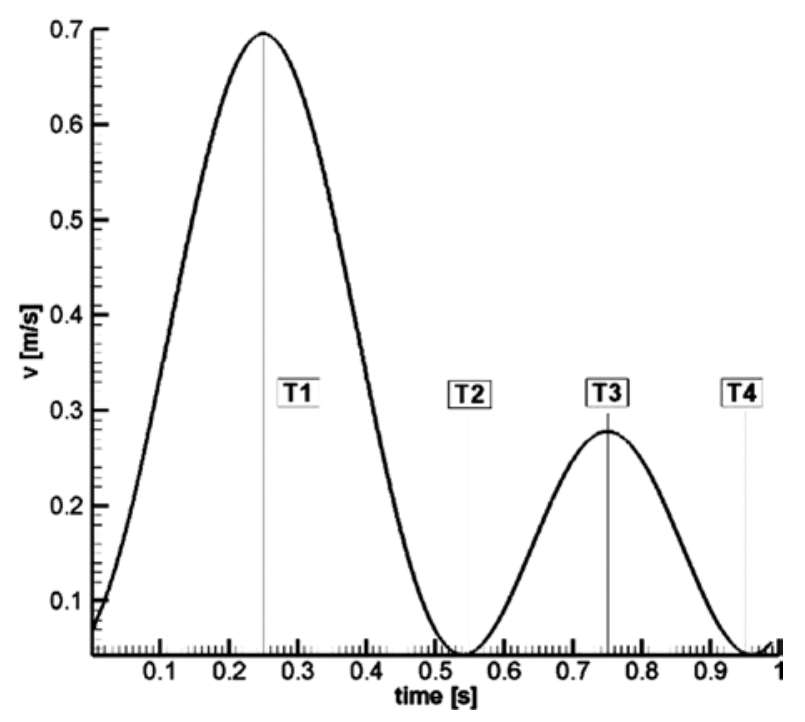

Figure 4: Pulsatile inlet flow waveforms throughout one cardiac cycle used as the input function at the RCA for case 1 (eqn (1)).
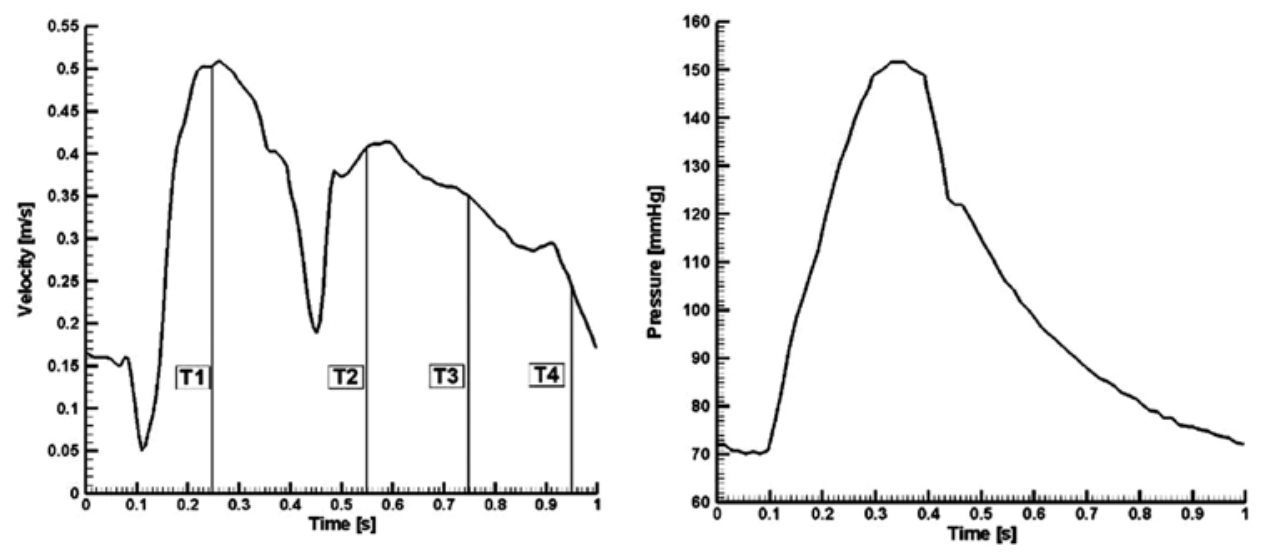

Figure 5: Velocity (left) used as the input function for case 2 and pressure (right) used as the outlet functions, throughout one cardiac cycle for patient with stenosed RCA. Values are based on measurements from the literature [26]. Reference phases at which results are presented are also indicated. The reference phases are the same for both the cases. 
Comparisons between the two input wave models are presented in terms of hemodynamic factors (flow patterns, flow velocity, WSS distributions), as it is believed that WSS is a significant factor in the onset of coronary artery disease [27, 28].

For both cases a straight entrance length of 10 inlet cross-section diameters was included at the inlet to avoid the effect of inlet velocity profile on the fluid dynamics at the region of interest [29].

The velocity boundary condition was always specified normal to the inlet cross-section. Five diameter extensions on the downstream side was sufficient to avoid the effect of the outlet zero pressure boundary condition (for case 1) on the fluid dynamics in the stenosed region. For case 2 at the exit section of the outlet extension a physiologically realistic pressure waveform was applied.

Each extension consisted of a straight section that connected the reconstructed RCA geometry with the fixed straight section. Care was taken to ensure that there were smooth connections between the extension sections and the RCA geometry. The influence of the boundary condition on the solution in the region of interest was minimal.

\section{RESULTS}

In this work we modeled blood flow in a stenosed RCA using two different inlet waveforms (a physiologically realistic inlet velocity profile, and one realistic approximation of the physiologic waveforms described by the mean and two harmonic terms, eqn (1)).

Our goal was to quantify the importance of the inlet velocity waveforms for the RCA hemodynamics, in order to guide further modeling studies and to assess the potential importance of the input boundary condition for investigation and the validation of causal links between hemodynamic flow variables and coronary artery pathology.

The RCA is a common site of atherosclerosis, which is linked to hemodynamic factors such as low WSS $[11,26]$. The effects of the difference in blood flow waveforms on coronary hemodynamics in RCA were investigated using CFD.

Transient simulations were performed for the stenosed RCA artery described above. Each simulation was from $t=0$ to $1.00 \mathrm{~s}$, yielding a heart rate of approximately 64 beats per minute. For case 1 , the cardiac cycle period was $1 \mathrm{~s}$, with peak systolic flow occurring at $0.25 \mathrm{~s}$, and peak diastolic flow at $0.75 \mathrm{~s}$. The lengths of the systolic and diastolic phases were 0.6 and $0.4 \mathrm{~s}$, respectively. For case 2, the cardiac cycle period is the same as for case 1 , with peak systolic flow occurring at $0.27 \mathrm{~s}$, and peak diastolic flow at $0.59 \mathrm{~s}$. The lengths of the systolic and diastolic periods were 0.34 and $0.66 \mathrm{~s}$, respectively.

Representative data set of the hemodynamics parameters in stenosed RCA are shown at the time values of 0.25 and $0.75 \mathrm{~s}$ through the cardiac cycle. These values were chosen as they are representative during the cardiac cycle: $t=0.35 \mathrm{~s}$ is at the peak of the systolic phase, and $t=0.75 \mathrm{~s}$ corresponds to the middle point of the diastolic phase.

\subsection{Flow separation and secondary flow}

The natural curvature of the RCA is expected to induce significant secondary flow motion or crossflow motion within the artery. RCA curvature gradually changes the flow in sections D0, D3, D5, D7. Downstream of these sections, the flow is highly three-dimensional, being sharply skewed toward of the RCA wall.

In the spacing between succesive constrictions (between: sections D2 and D4; sections D4 and D6; sections D6 and D8) the flow repeatedly undergoes an expansion after each constriction where the separation zone formed in sections D3, D5 and D6 (Fig. 6). Energy loss associated with such flow expansion after each constriction will be large and consequently the pressure drop will be higher (Tables 5 and 6). 
Artery section constriction and post-constriction dilatations have led to acceleration and rapid deceleration, respectively, including a distortion of flow. Large recirculation regions found in the vicinity of the each constricted section (Fig. 6).

Data corresponding to the velocity field obtained in the reconstructed RCA for both input velocity profiles is presented in Table 3 (at the investigated time $\mathrm{T} 1=0.25 \mathrm{~s}$ ) and Table 4 (at the investigated time T4 $=0.75 \mathrm{~s}$ ). Good agreement has been demonstrated between coronary blood
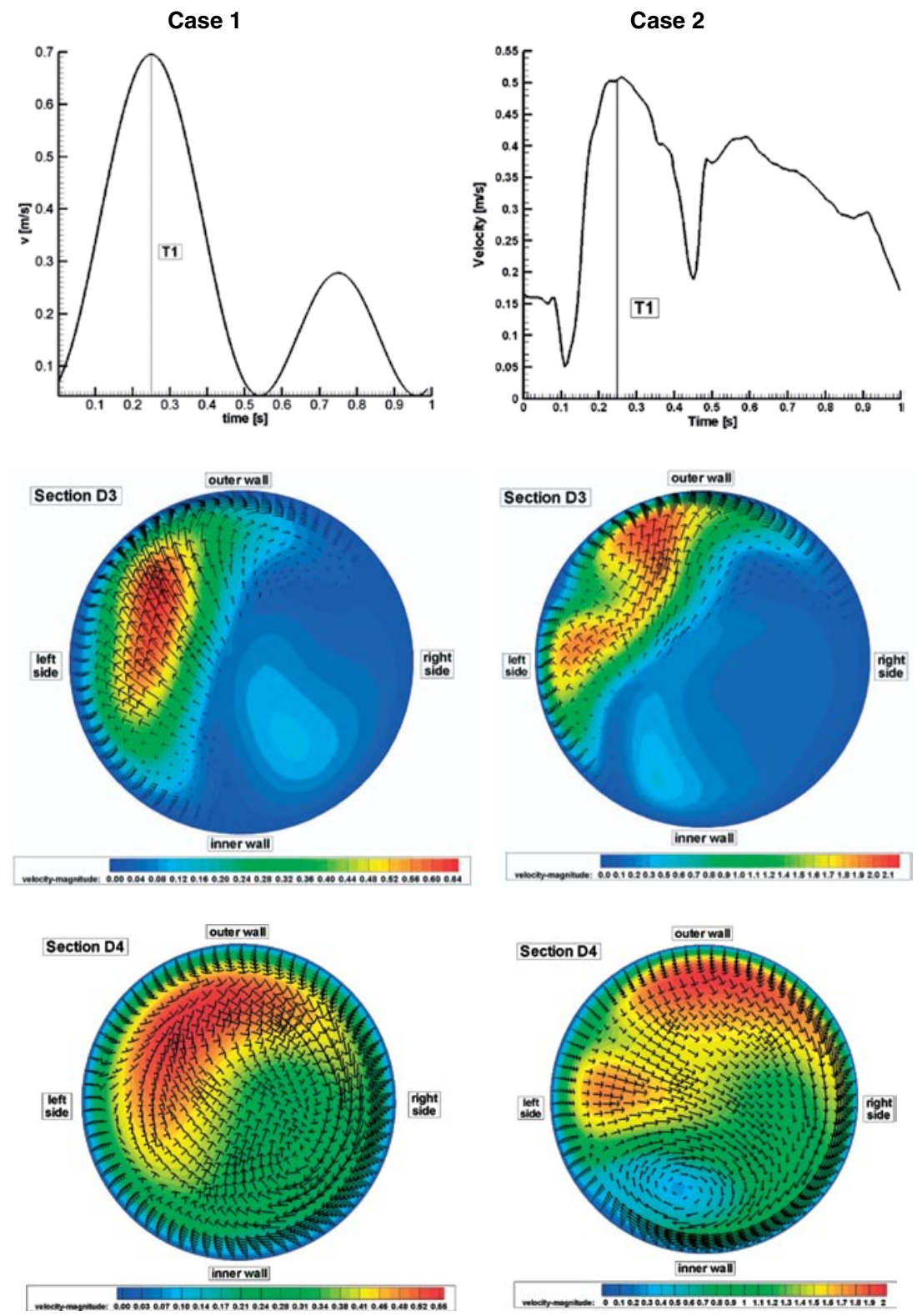

Figure 6: Transverse velocity profiles of the blood flow in stenosed RCA, in selected cross-sections for both input velocity waveforms at the time $\mathrm{T} 1=0.25 \mathrm{~s}$. 

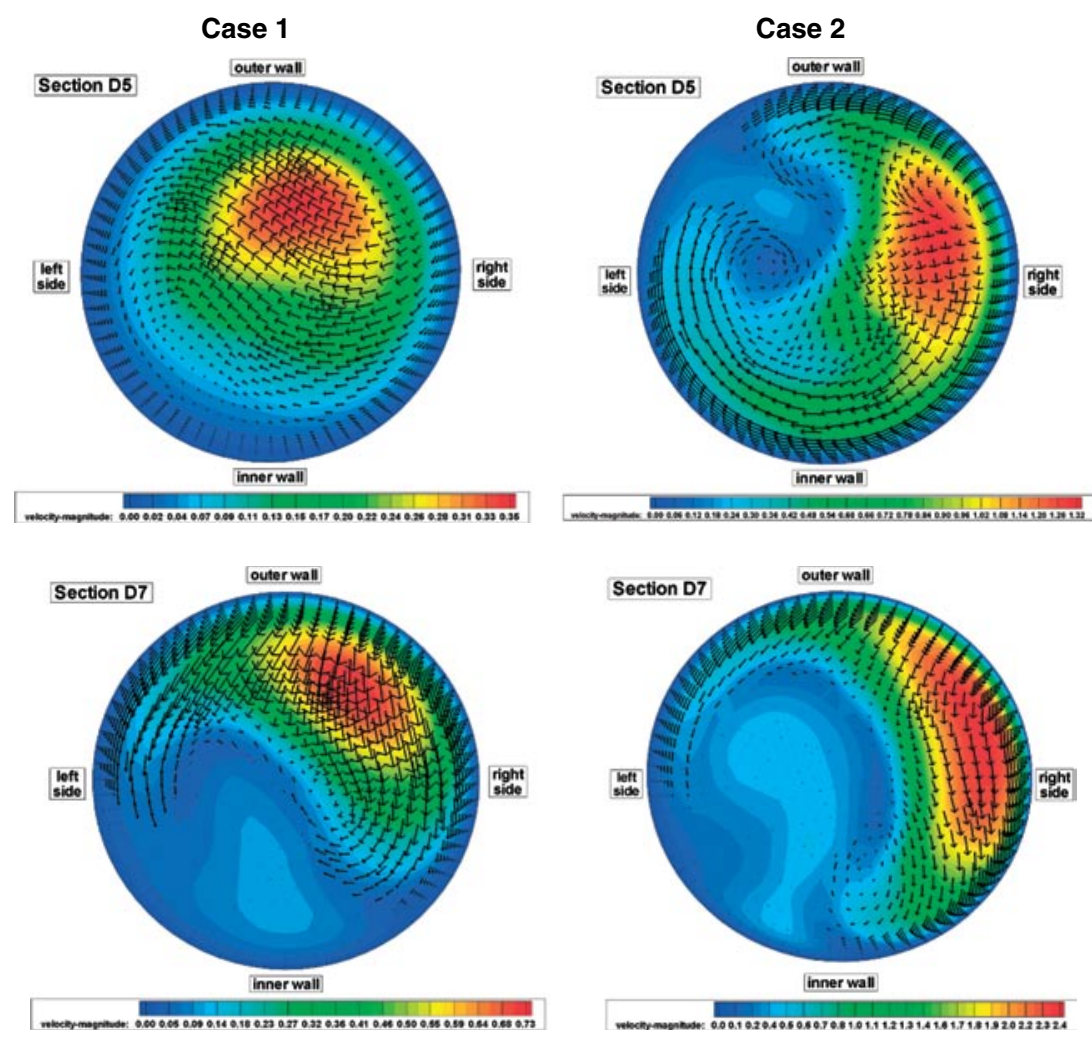

Figure 6: (continued).

Table 3: Blood velocities analysis in the cross-sections of interest defined in Fig. 2 at the time $\mathrm{T} 1=0.25 \mathrm{~s}$.

\begin{tabular}{|c|c|c|c|c|c|c|}
\hline \multirow[b]{3}{*}{ Section } & \multicolumn{4}{|c|}{ Time T1 } & \multirow{2}{*}{\multicolumn{2}{|c|}{ Percent difference }} \\
\hline & \multicolumn{2}{|c|}{ Case 1} & \multicolumn{2}{|c|}{ Case 2} & & \\
\hline & $\mathrm{Vc}(\mathrm{m} / \mathrm{s})$ & $\operatorname{Vav}(\mathrm{m} / \mathrm{s})$ & $\mathrm{Vc}(\mathrm{m} / \mathrm{s})$ & $\operatorname{Vav}(\mathrm{m} / \mathrm{s})$ & $\mathrm{Vc}(\%)$ & $\operatorname{Vav}(\%)$ \\
\hline D0 & 0.281 & 0.15 & 0.236 & 0.505 & 19 & 70.3 \\
\hline D1 & 0.859 & 0.69 & 0.905 & 2.338 & 5 & 70.3 \\
\hline D2 & 0.792 & 0.66 & 0.915 & 2.259 & 13.4 & 70.6 \\
\hline D3 & 0.061 & 0.15 & 0.146 & 0.550 & 58.2 & 72.7 \\
\hline D4 & 0.361 & 0.32 & 0.397 & 1.153 & 9 & 72.1 \\
\hline D5 & 0.253 & 0.12 & 0.201 & 0.470 & 25.8 & 74.4 \\
\hline D6 & 1.043 & 0.72 & 1.103 & 2.450 & 5.4 & 70.6 \\
\hline D7 & 0.100 & 0.21 & 0.419 & 0.780 & 76.13 & 73 \\
\hline D8 & 0.995 & 0.84 & 1.079 & 2.870 & 7.7 & 70.7 \\
\hline
\end{tabular}

$\mathrm{Vc}$ - velocity magnitude in the center of the investigated sections.

Vav - area weighted average velocity magnitude in the cross-sections of interest. 
Table 4: Blood velocities analysis in the cross-sections of interest at the time $\mathrm{T} 4=0.75 \mathrm{~s}$.

\begin{tabular}{|c|c|c|c|c|c|c|}
\hline \multirow[b]{3}{*}{ Section } & \multicolumn{6}{|c|}{ Time T4 } \\
\hline & \multicolumn{2}{|c|}{ Case 1} & \multicolumn{2}{|c|}{ Case 2} & \multicolumn{2}{|c|}{ Percent difference } \\
\hline & $\mathrm{Vc}(\mathrm{m} / \mathrm{s})$ & $\operatorname{Vav}(\mathrm{m} / \mathrm{s})$ & $\mathrm{Vc}(\mathrm{m} / \mathrm{s})$ & $\operatorname{Vav}(\mathrm{m} / \mathrm{s})$ & $\mathrm{Vc}(\%)$ & $\operatorname{Vav}(\%)$ \\
\hline D0 & 0.113 & 0.0096 & 0.589 & 0.349 & 80.8 & 97.2 \\
\hline D1 & 0.381 & 0.0447 & 1.863 & 1.618 & 79.5 & 97.2 \\
\hline D2 & 0.396 & 0.0429 & 1.673 & 1.561 & 76.3 & 97.2 \\
\hline D3 & 0.033 & 0.0140 & 0.171 & 0.379 & 80.7 & 96.3 \\
\hline D4 & 0.178 & 0.0209 & 1.003 & 0.788 & 82.2 & 97.3 \\
\hline D5 & 0.118 & 0.0083 & 0.104 & 0.317 & 13.4 & 97.3 \\
\hline D6 & 0.512 & 0.0461 & 2.020 & 1.681 & 74.6 & 97.2 \\
\hline D7 & 0.148 & 0.0151 & 0.221 & 0.529 & 33 & 97.1 \\
\hline D8 & 0.455 & 0.0547 & 2.103 & 1.985 & 78.3 & 97.2 \\
\hline
\end{tabular}

$\mathrm{Vc}$ - velocity magnitude in the center of the investigated sections.

Vav - area weighted average velocity magnitude in the cross-sections of interest.

Table 5: Pressure variation in the sections of interest defined in Fig. 2 at the time $\mathrm{T} 1=0.25 \mathrm{~s}$.

\begin{tabular}{|c|c|c|c|c|c|c|}
\hline \multirow[b]{3}{*}{ Section } & \multicolumn{6}{|c|}{ Time T1 } \\
\hline & \multicolumn{2}{|c|}{ Case 1} & \multicolumn{2}{|c|}{ Case 2} & \multicolumn{2}{|c|}{ Percent difference } \\
\hline & $\mathrm{Pc}(\mathrm{mmHg})$ & Pav (mmHg) & $\mathrm{Pc}(\mathrm{mmHg})$ & Pav (mmHg) & $\operatorname{Pc}(\%)$ & $\operatorname{Pav}(\%)$ \\
\hline D0 & 28.21 & 28.22 & 324 & 324.47 & 91.29 & 91.30 \\
\hline D1 & 25.49 & 25.39 & 299 & 298.13 & 91.47 & 91.48 \\
\hline D2 & 24.04 & 23.81 & 290 & 287.69 & 91.71 & 91.72 \\
\hline D3 & 24.38 & 24.42 & 291 & 292.56 & 91.62 & 91.65 \\
\hline D4 & 24.32 & 24.24 & 292 & 291.81 & 91.67 & 91.69 \\
\hline D5 & 24.41 & 24.43 & 293 & 293.78 & 91.67 & 91.68 \\
\hline D6 & 21.38 & 20.63 & 260 & 260.87 & 91.78 & 92.09 \\
\hline D7 & 21.04 & 21.05 & 264 & 264.40 & 92.03 & 92.04 \\
\hline D8 & 17.58 & 17.45 & 234 & 232.68 & 92.49 & 92.50 \\
\hline
\end{tabular}

$\mathrm{Pc}-$ pressure in the center of the investigated sections.

$\mathrm{Pav}-$ area weighted average pressure in the investigated sections.

velocity obtained in present work using input velocity described in case 2 and the measurements presented by Siebes et al. [30].

\subsection{Pressure}

As the physiological pressure conditions were applied at the outlet for case 2 and pressure level 0 is selected at the outlet in case 1 .

We selected the reference pressure as the pressure at the outlet in case 2 in order to estimate the percentage difference between the pressure variations in both cases, and to better compare the pressure drop under the effect of different sizes of stenosis.

To understand the pressure-flow relationship, pressure variations across the different sections of the RCA have been evaluated. In Fig. 7 the pressure field drop along the RCA has been plotted covering the total investigated length of the RCA. 
Table 6: Pressure variation in the cross-sections of interest defined in Fig. 2 at the time T4=0.75 s.

\begin{tabular}{|c|c|c|c|c|c|c|}
\hline \multirow[b]{3}{*}{ Section } & \multicolumn{6}{|c|}{ Time T4 } \\
\hline & \multicolumn{2}{|c|}{ Case 1} & \multicolumn{2}{|c|}{ Case 2} & \multicolumn{2}{|c|}{ Percent difference } \\
\hline & $\mathrm{Pc}(\mathrm{mmHg})$ & $\mathrm{Pav}(\mathrm{mmHg})$ & $\mathrm{Pc}(\mathrm{mmHg})$ & Pav (mmHg) & $\mathrm{Pc}(\%)$ & $\operatorname{Pav}(\%)$ \\
\hline D0 & 8.78 & 8.78 & 191 & 191.42 & 95.41 & 95.42 \\
\hline D1 & 8.21 & 8.20 & 178 & 178.25 & 95.39 & 95.40 \\
\hline D2 & 7.79 & 7.75 & 173 & 172.61 & 95.50 & 95.51 \\
\hline D3 & 7.85 & 7.85 & 174 & 175.22 & 95.49 & 95.52 \\
\hline D4 & 7.79 & 7.79 & 175 & 174.79 & 95.55 & 95.55 \\
\hline D5 & 7.79 & 7.80 & 175 & 175.71 & 95.55 & 95.56 \\
\hline D6 & 7.14 & 6.97 & 159 & 159.27 & 95.51 & 95.62 \\
\hline D7 & 7.05 & 7.05 & 161 & 160.90 & 95.62 & 95.62 \\
\hline D8 & 6.29 & 6.26 & 145 & 144.69 & 95.67 & 95.67 \\
\hline
\end{tabular}

$\mathrm{Pc}-$ pressure in the center of the investigated sections.

$\mathrm{Pav}-$ area weighted average pressure in the investigated sections.
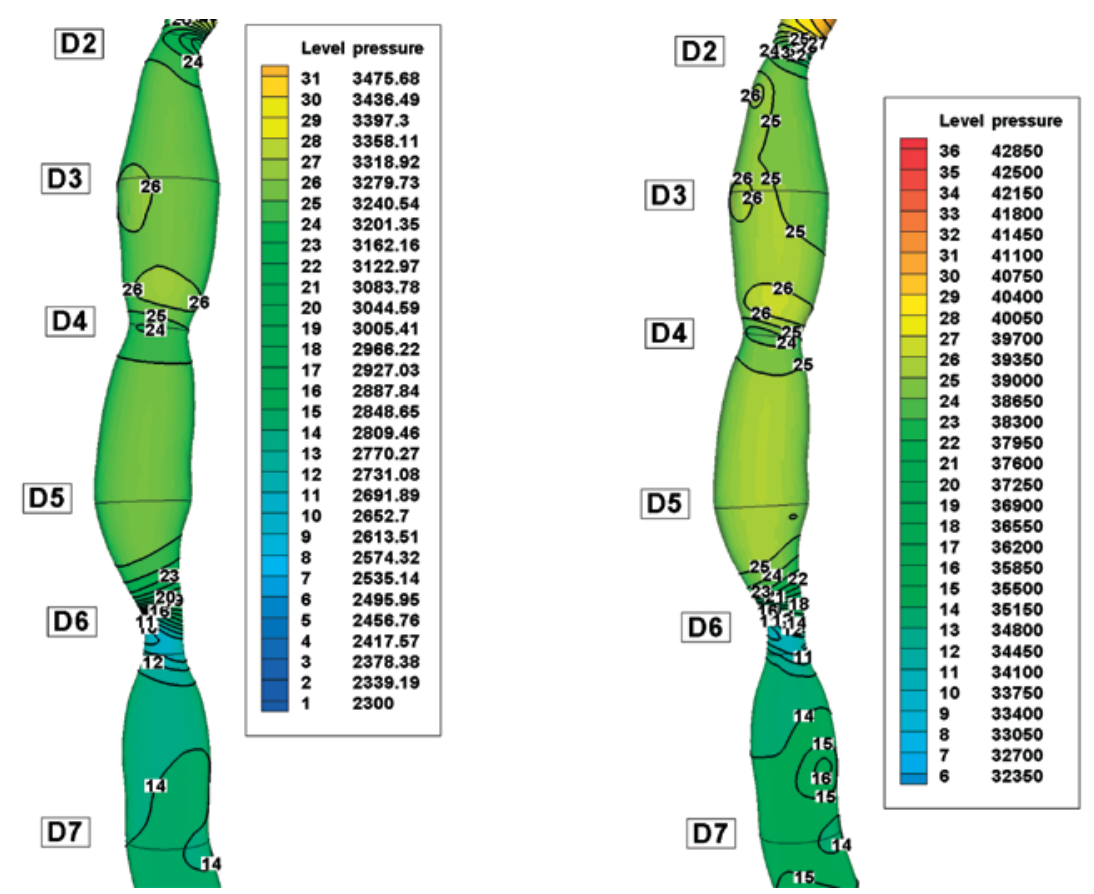

Figure 7: Pressure field (Pa) in stenosed RCA (Fig. 2) for both input velocity waveforms at the investigated time $\mathrm{T} 1=0.25 \mathrm{~s}$.

The knowledge of local pressure becomes mandatory when fluid structure interactions between the fluid and the vessel wall need to be considered. It would thus appear necessary to quantify the accurate pressure profiles within the coronary (Fig. 7).

The main function of wall pressure representing a map of the pressure inside the coronary artery is to push the blood into the capillaries and facilitate diffusion into the myocardium. For the patient 
of this study, the wall pressure decreased towards the periphery of the coronary artery with elevated pressure drops in stenotic segments. The increased pressure drop in stenoses reflects the elevated energy needed to drive the flow through these regions.

\subsection{Wall shear rates}

Wall shear stress and pressure are likely the most relevant parameters from the standpoint of the fluid mechanical involvement in the development of atherosclerosis.

The fluid-induced WSS is a very small frictional force induced by a viscous fluid flow moving on the surface of solid materials. The endothelial cell is capable of sensing the level and the direction of the WSS induced by the blood flow, and the biomechanical properties of endothelial cell are partially regulated by the WSS $[2,4]$. The endothelial dysfunction induced by a certain hemodynamic condition is an elemental feature in the formation of atherosclerotic plaque. It is widely believed that atherosclerosis development and progression are affected by many risk factors, such as static pressure, WSS, blood viscosity, flow velocity, etc.

The magnitude and direction of WSS influence inflammatory processes in the vessels. Although the interaction of WSS and inflammation involves a complicated cascade, slow and turbulent flow condition has been shown to induce gene expression of proinflammatory molecules in the endothelial cells $[9,10]$.

The shear rate $(\gamma(1 / \mathrm{s}))$ for a Newtonian one-directional flow in a cylindrical tube is equal to the velocity gradient with respect to radial position:

$$
\gamma=-\frac{d v}{d r}
$$

where $v(\mathrm{~m} / \mathrm{s})$ is the axial velocity and $r(\mathrm{~m})$ is the radial distance from the center of the vessel. The SS is the force per unit area applied to a fluid layer producing its movement relative to the adjacent layers and is equal to the product of shear rate and the viscosity:

$$
\tau=\mu \cdot \gamma=-\mu \frac{d v}{d r}
$$

Because the vessel narrows at the stenosis, the velocity at the center of the vessel increases due to the conservation of mass law. The velocity gradient at the wall and the resulting SS is maximum at the narrowest portion of the stenosis (Fig. 8). Along the stenosed arterial wall, the SS varies significantly from point to point. The SS, very low outside the stenosis, suddenly increases with the narrowing diameter, and reaches a maximum at the smallest diameter inside the stenosis (Fig. 8). Increasing the amount of stenosis usually reduced the flow through the stenosis. However, due to the cubic dependence of shear on diameter, the overall effect is to increase the SS.

Parallel to the changes of WSS, the flow pattern was more variable and inconsistent in the vicinity of the constriction (Tables 7 and 8). Artery section constriction and post-constriction dilatations have led to acceleration and rapid deceleration, respectively, including a flow distortion.

\section{DISCUSSION}

\subsection{Flow separation}

Various patterns of transverse velocity fields at radial cross-sections demonstrated a significant secondary flow in the curved arteries and a strong effect of stenosis on this flow dynamic phenomenon. 

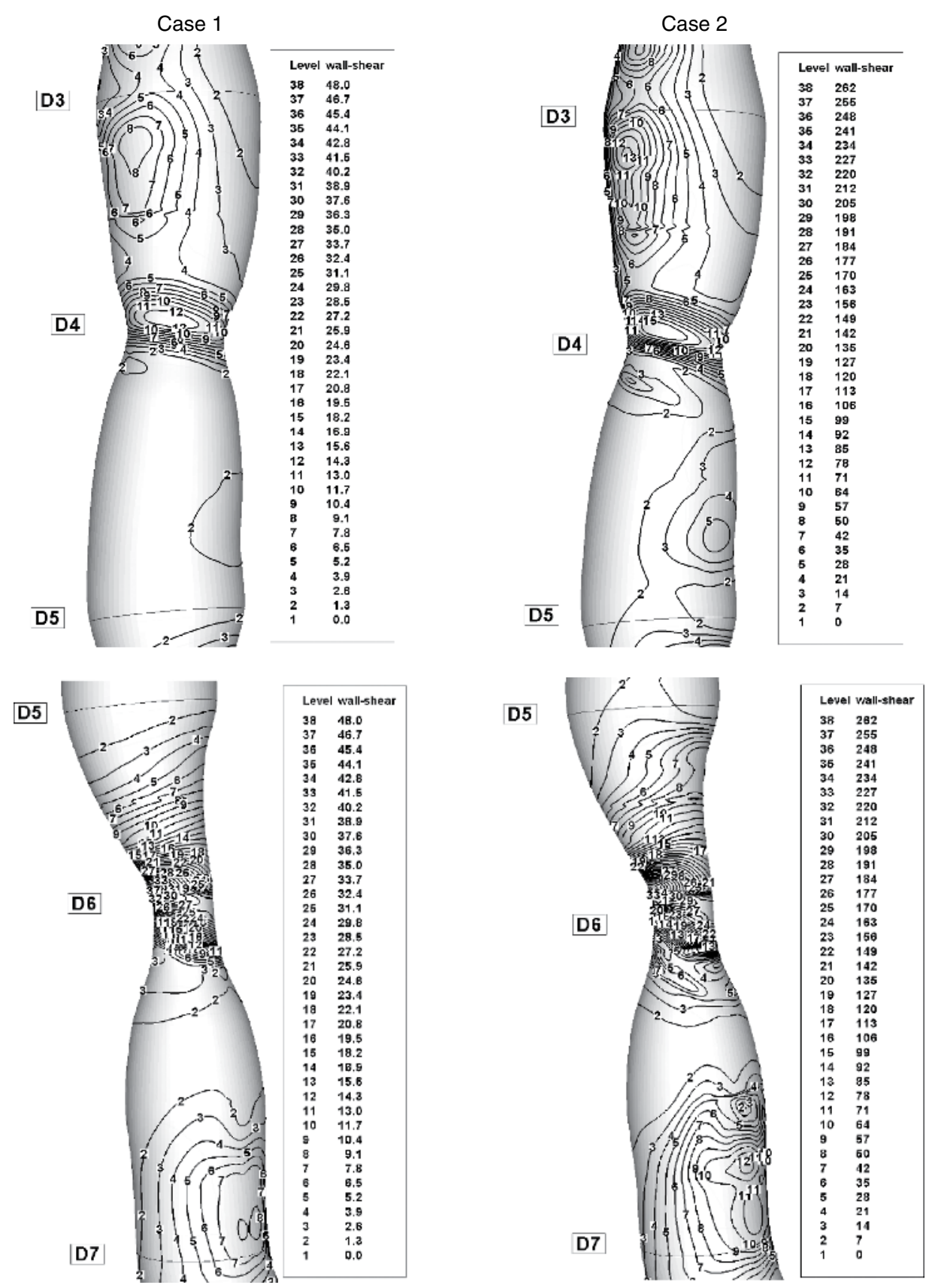

Figure 8: Contour plot of WSS magnitude in the sections of interest defined at the time T1 $=0.25 \mathrm{~s}$ : case 1 (left column); case 2 (right column). 
Table 7: Minimum/maximum shear rates $(\mathrm{Pa})$ in the cross-sections (time $\mathrm{T} 1=0.25 \mathrm{~s}$ ).

\begin{tabular}{|c|c|c|c|c|}
\hline \multirow[b]{3}{*}{ Section } & \multicolumn{4}{|c|}{ Time T1 } \\
\hline & \multicolumn{2}{|c|}{ Case 1} & \multicolumn{2}{|c|}{ Case 2} \\
\hline & WSS min $(\mathrm{Pa})$ & WSS max $(\mathrm{Pa})$ & WSS min $(\mathrm{Pa})$ & WSS max $(\mathrm{Pa})$ \\
\hline D0 & 2.00 & 4.00 & 7 & 14.00 \\
\hline D1 & 26.00 & 31.00 & 140 & 168.00 \\
\hline D2 & 12.00 & 34.00 & 63 & 133.00 \\
\hline D3 & 1.00 & 2.00 & 7 & 49.00 \\
\hline D4 & 6.00 & 15.00 & 21 & 91.00 \\
\hline D5 & 1.00 & 2.00 & 7 & 9.00 \\
\hline D6 & 4.00 & 42.00 & 154 & 238.00 \\
\hline D7 & 2.00 & 4.00 & 7 & 56.00 \\
\hline D8 & 38.00 & 44.00 & 196 & 231.00 \\
\hline
\end{tabular}

Table 8: Minimum/maximum shear rates $(\mathrm{Pa})$ in the cross-sections (time $\mathrm{T} 4=0.75 \mathrm{~s}$ ).

\begin{tabular}{|c|c|c|c|c|}
\hline \multirow[b]{3}{*}{ Section } & \multicolumn{4}{|c|}{ Time T4 } \\
\hline & \multicolumn{2}{|c|}{ Case 1} & \multicolumn{2}{|c|}{ Case 2} \\
\hline & WSS min $(\mathrm{Pa})$ & WSS max $(\mathrm{Pa})$ & WSS min $(\mathrm{Pa})$ & WSS max (Pa) \\
\hline D0 & 0.50 & 0.80 & 4 & 5.00 \\
\hline D1 & 6.30 & 8.50 & 88 & 100.00 \\
\hline D2 & 3.80 & 11.00 & 36 & 124.00 \\
\hline D3 & 0.40 & 0.50 & 4 & 28.00 \\
\hline D4 & 2.00 & 3.00 & 24 & 52.00 \\
\hline D5 & 0.20 & 0.30 & 4 & 8.00 \\
\hline D6 & 8.00 & 11.80 & 100 & 148.00 \\
\hline D7 & 0.30 & 1.30 & 4 & 32.00 \\
\hline D8 & 10.00 & 11.80 & 132 & 140.00 \\
\hline
\end{tabular}

It was found that the secondary flow became stronger and the pattern varied irregularly in the downstream curved stenotic arteries due to the presence of a stenosis (Fig. 6).

Stenoses and post-stenotic dilatations have led to acceleration and rapid deceleration, respectively, including a distortion of flow. Large recirculation regions found in the vicinity of the each constricted section. Post-stenotic deceleration of blood flow induces flow separation and recirculation zones (vortices). Such regions of flow separation and statis are favored sites for the development of thrombosis and atherosclerosis [8]. A closer inspection on these patterns reveals that complex recirculation zones develop in the vicinity of the sections D3, D5 and D7 of the RCA during systolic phase of both types of the input waveforms (Fig. 6). Appearance of these recirculation zones coincides with inflow deceleration during that period of the cardiac cycle. These particular areas coincide with the regions of minimum WSS (Fig. 8).

\subsection{Pressure}

Pressure wave propagation and reflection are not incorporated in the present model. In spite of that, the current approach is valid in the branchless RCA model given the expected long pressure wavelengths. 
In patients with multiple stenoses within the same coronary artery, coronary pressure investigation can uniquely determine the separate hemodynamic effects of the individual stenoses in sequence [31]. For clinical practice, pressure recording at maximum hyperemia provides important information, which can help objectively select which of several stenoses is most appropriate for PTCA. This information will also allow clinicians to avoid performing unnecessary procedures that increase the risk of restenosis without a hemodynamic benefit.

\subsection{Wall shear stress}

Wall shear stress is another important parameter in the study of stenotic flow. Gibson et al. [11] investigated the relation between vessel WSS and the rate of atherosclerosis progression by means of quantitative angiography. Their results showed that there was a significant correlation between low shear stress and an increased rate of atherosclerosis progression.

The change of WSS throughout the cardiac cycle highly correlated to flow velocities. The WSS characterizes the forces that longitudinally act on the vessel wall. These forces were high when the blood flow parallel to the wall was fast. The maximum WSS spatial variation was approximately 46 $\mathrm{Pa}$ in stenosed sections D4 and D6 (Fig. 8 and Table 7) whereas the corresponding maximum wall pressure drop reached $460 \mathrm{~Pa}$, for case 1 at the time $\mathrm{T} 1=0.25 \mathrm{~s}$. For case 2 , the WSS maximum spatial variation is $260 \mathrm{~Pa}$ in sections D4 and D6, corresponding to the maximum pressure drop reached $1600 \mathrm{~Pa}$.

\subsection{Study limitations}

Some limitations of our study should be pointed out. First, the heart movement and the movement of the coronary arteries due to muscle tension cannot be simulated yet, therefore, not included in the present calculations. Second, we modeled only the trunk of the RCA by ignoring all of its branches. Third, in this model vessel walls are assumed rigid.

Zeng et al. [32] have incorporated the effects of physiologically realistic arterial motion into a simulation of blood flow patterns in the RCA. Our results show that arterial motion had little effect on the WSS distribution within the RCA, and that flow in the moving artery followed the instantaneous dynamic geometry quite closely. These results agree with the findings of other groups [33, 34].

Localized high stresses in the vessel wall have been found to relate to local disease development [35]. Furthermore, it has been indicated by numerous studies that fluid dynamics plays an important role in the initiation and development of atherosclerosis $[36,37]$ and it is possible that certain arterial motion may promote atherosclerosis by creating or exacerbating a pathogenic fluid dynamic environment.

\section{CONCLUSION}

In the work described here, we compared the fluid dynamics for the same arterial segment using two different input pulsatile conditions.

Employing a pulsatile flow curve from the literature, we compared the computed axial velocity distribution, WSS and pressure distribution at selected cross-sections, between both input waveforms investigated. We therefore conclude that the effect of simplification of the real inlet velocity profile in the RCA will significantly affect conclusions drawn from the hemodynamic parameters of WSS, pressure distribution, velocity field and secondary flow development, in realistic models of the human stenosed RCA. However, it is conceptually important to realize that one stenosis influences the hemodynamic effects of another in a sequence that may result in a mutual underestimation of the severity of each unless stenosis interaction is accounted for. 
The study also provides awareness that the presence of one stenosis in a coronary artery influences the hemodynamic appearance of the other and, consequently, that treating one lesion will unmask the true severity of the second. As shown in this study, it is possible to calculate this effect quantitatively by measuring pressures at the relevant sites within the artery.

The influence of a distal stenosis on the hemodynamic appearance of a proximal stenosis is generally larger than that of a proximal stenosis on a distal one (Tables 3-6).

The fluid, dynamic interaction of multiple sequential stenoses in coronary arteries is complex, often unexpected, and cannot be adequately assessed by visual interpretation on the coronary angiogram. Assessment of shear stress, along with percent stenosis, in arterial lesions might prove to be a valuable diagnostic tool to identify patients at risk of developing acute platelet thrombus formation in stenosed arteries. Computer flow models can be utilized in conjunction with human coronary angiography to study flow characteristics for plaques of different sizes and shapes in patients with stable and unstable angina.

Wall shear stress and its interaction with the endothelial cell and smooth muscle cell regulate not only the vessel diameter but also the proliferation of endothelial and smooth muscle cells, endothelial permeability, and inflammation in the vessels. Laminar and physiologic shear stress is one of the crucial factors to maintain the proper level of NO, a key mediator of vessel behavior. Attenuation of $\mathrm{NO}$ is one of the earliest changes preceding endothelial dysfunction. Endothelial dysfunction induced by slow or turbulent flow condition promotes atherosclerosis in conjunction with such systemic risk factors as hypercholesteremia, hypertension, diabetes, and smoking.

\section{ACKNOWLEDGMENTS}

The present research has been supported by the Romanian National Authority for Scientific Research through the CNCSIS 798/2008 project, contract no: 590/2009.

\section{REFERENCES}

[1] Buchanan, J.R. \& Kleinstreuer, C., Simulation of particle-hemodynamics in a partially occluded artery segment with implications to the initiation of microemboli and secondary stenoses. Journal of Biomechanical Engineering, 120, pp. 446-454, 1998. doi:10.1115/1.2798013

[2] Ciu, J.-J., Wang, D.L., Chien, S., Skalak, R. \& Usami S., Effects of disturbed flow on endothelial cells. Journal of Biomechanical Engineering, 120, pp. 2-8, 1998. doi:10.1115/1.2834303

[3] Marsahall, I., Zhao, S., Papathanasopoulou P., Hoskins P. \& Yun Xu X., MRI and CFD studies of pulsatile flow in healtly and stenosed carotid bifurcation models. Journal of Biomechanics, 37, pp. 679-687, 2004. doi:10.1016/j.jbiomech.2003.09.032

[4] Sun, N., Torii R., Wood, N.B., Hughes A.D., Thom S.A.M., \& Xu Y.X., Computaional modeling of LDL and albumin transport in an in vivo CT image-based human right coronary artery. Journal of Biomechanical Engineering, 131, pp. 1-9, 2009. doi:10.1115/1.3005161

[5] Frauenfelder, T., Boutsianis, E., Schertler T., et al., In-vivo flow simulation in coronary arteries based on computed tomography datasets: feasibility and initial results. Eur Radiol, 17(5), pp. 1291-1300, 2007. doi:10.1007/s00330-006-0465-1

[6] Liu, B., The influences of stenosis on the downstream flow pattern in curved arteries. Medical Enginnering \& Physics, 29, pp. 868-876, 2007. doi:10.1016/j.medengphy.2006.09.009

[7] Sherwin, S.J. \& Blackburn, HM., Three-dimensional instabilities and transition of steady and pulsatile axisymmetric stenotic flows. Journal Fluid Mechanics, 533, pp. 297-327, 2005. doi:10.1017/S0022112005004271

[8] Dodds, S.R., The haemodynamics of asymmetric stenoses. European Journal of Vascular Endovascular Surgery, 24, pp. 332-337, 2002. doi:10.1053/ejvs.2002.1729 
[9] Zeng, D., Boutsianis, E., Ammann, M., Boomsma, K., Wildermuth, S. \& Poulikakos, D., A study of the compliance of a right coronary artery and its impact on wall shear stress. Journal of Biomechanical Engineering, 130, pp. 041014-11, 2008. doi:10.1115/1.2937744

[10] Truskey, G.A., Barber, K.M., Robey, T.C., Lauri, A.O. \& Combs, M.P., Characterization of a sudden expansion flow chamber to study the response of endothelium to flow recirculation. Journal of Biomechanical Engineering, 117, pp. 203-210, 1995. doi:10.1115/1.2796002

[11] Gibson, C.M., Diaz, L., Kandarpa, K., et al., Relation of vessel wall shear stress to atherosclerosis progression in human coronary arteries. Arteriosclerosis Thrombosis, 13(2), pp. 310-315, 1993.

[12] Tateshima, S., Tanishita, K. \& Vinuela, F., Hemodynamics and cerebrovascular disease. Surgical Neurology, 70, pp. 447-453, 2008. doi:10.1016/j.surneu.2008.07.010

[13] Hyun, S., Kleinstreuer, C. \& Archie, J.P. Jr, Computational particle-hemodynamics analysis and geometric reconstruction after carotid endarterectomy. Computers in Biology and Medicine, 31, pp. 365-384, 2001. doi:10.1016/S0010-4825(01)00007-5

[14] Spicer, S.A. \& Taylor, C.A., Simulation ased Medical Planning for Cardiovascular Disease: Visualization System Foundations. Computer Aided Surgery, 5, pp. 82-89, 2000. doi:10.3109/10929080009148874, doi:10.1002/1097-0150(2000)5:2<82::AID-IGS2>3.3.CO;2-X

[15] Bernad, S.I., Bernad, E. \& Mihalas, G.I., Numerical investigation of blood flow in the arterial stenosis, Proc. of the Medical Informatics Europe - MIE2003, eds Baund, R., Fieschi, M., Le Beux, P.\& Ruch, P., IOS Press: Amsterdam, The Netherlands, pp. 3-8, 2003.

[16] Rathish Kumar, B.V., Yamaghuchi, T., Liu, H. \& Himeno, R., A numerical study of an unsteady laminar flow in a doubly constricted 3D vessel. International Journal for Numerical Methods in Fluids, 38, pp. 1159-1176, 2002. doi:10.1002/fld.191

[17] Shahcheraghi, N., Dwyer, H.A., Cheer, A.Y., Barakat A.I. \& Rutaganira T., Unsteady and treedimensional simulation of blood flow in the human aortic arch. Journal of Biomechanical Engineering, 124, pp. 378-387, 2002. doi:10.1115/1.1487357

[18] Yung, C.N., De Witt, K.J., Subramanian, S., Afjeh, A.A. \& Keith, T.G., Three-dimensional pulsatile flow through a bifurcation. International Journal of Numerical Methods for Heat \& Fluid Flow, 7(8), pp. 843-862, 1997. doi:10.1108/09615539710193010

[19] Eliasziw, M., Smith, R.F., Singh, N., et al., Further comments on the measurement of carotid stenosis from angiograms: North American Symptomatic Carotid Endarterectomy Trial (NASCET) Group. Stroke, 25, pp. 2445-49, 1994.

[20] Rothwell, P.M., Eliasziw, M., Gutnikov, S.A., et al., Analysis of pooled data from the randomized controlled trials of endarterectomy for symptomatic carotid stenosis. Lancet, 361, pp. 107-16, 2003. doi:10.1016/S0140-6736(03)12228-3

[21] Kiviniemi, T.O., Saraste, M., Koskenvuo, J.W., et al., Coronary artery diameter can be assessed reliably with transthoracic echocardiography. American Journal Physiology. Heart Circulatory Physiology, 286, pp. H1515-H1520, 2004.

[22] FLUENT 6.3 User's Guide, Ansys Fluent Incorporated, 2006.

[23] Johnston, B.M., Johnston, P.R., Corney, S., Kilpatrick, D., Non-Newtonian blood flow in human right coronary arteries: transient simulations. Journal of Biomechanics, 39, pp. 1116-1128, 2005. doi:10.1016/j.jbiomech.2005.01.034

[24] Stroud, J.S., Berger, S.A. \& Saloner, D., Numerical analysis of flow through a severely stenotic carotid artery bifurcation. Journal of Biomechanical Engineering, 124, pp. 9-20, 2002. doi: $10.1115 / 1.1427042$

[25] Sherwin, S.J. \& Blackburn, H.M., Three-dimensional instabilities and transition of steady and pulsatile axisymmetric stenotic flows. Journal of Fluid Mechanics, 533, pp. 297-327, 2005. doi:10.1017/S0022112005004271 
[26] Torii, R., Wood, N.B., Hadjiloizou, N., Dowsey, A.W., Wright, A.R., Hughes, A.D., Davies, J., Francis, DP., Mayet, J., Yang, G-Z., Thom, S.A., \& Yun, X.X., Differences in coronary artery haemodynamics due to changes in flow and vascular geometry after percutaneous coronary intervention. Heart, 94, pp. A1-A4, 2008.

[27] Giddens, D.P., Zarins, C.K., \& Glagov, S., Response of arteries to near wall fluid dynamic behavior. Applied Mechanics Review, 43(2), pp. S98-S102, 1990.

[28] Ku, D., Giddens, D., Zarins, C., \& Glagov, S., Pulsatile flow and atherosclerosis in the human carotid bifurcation: positive correlation between plaque and low and oscillating shear stress. Arteriosclerosis, 5, pp. 293-302, 1985.

[29] Liu, Y., Lai, Y., Nagaraj, A., Kane, B., Hamilton, A., Greene, R., McPherson, D.D., \& Chandran, K. B., Pulsatile flow simulation in arterial vascular segments with intravascular ultrasound images. Medical Engineering\& Physics, 23(8), pp. 583-595, 2001. doi:10.1016/ S1350-4533(01)00088-1

[30] Siebes, M, Verhoeff, B-J., Meuwissen, M., de Winter, R.J., Spaan, J.A.E., \& Piek, J.J., Singlewire pressure and flow velocity measurement to quantify coronary stenosis hemodynamics and effects of percutaneous interventions. Circulation, 109, pp. 756-762, 2004. doi:10.1161/01. CIR.0000112571.06979.B2

[31] Pijls, N.H.J., De Bruyne, B., Jan Willem Bech, G., Liistro, F., Heyndrickx, G.R., Bonnier, H.J.R.M \& Koolen J.J., Coronary pressure measurement to assess the hemodynamic significance of serial stenoses within one coronary artery: validation in humans. Circulation, 102, pp. 2371-2377, 2000.

[32] Zeng, D., Ding, Z, Friedman, M.H., Ethier, C.R., Effects of cardiac motion on right coronary artery hemodynamics. Annals of Biomedical Engineering, 31, pp. 420-429, 2003. doi: $10.1114 / 1.1560631$

[33] Qiu, Y. \& Tarbell, J.M., Numerical simulation of pulsatile flow in a compliant curved tube model of a coronary artery. Journal of Biomechanical Engineering, 122, pp. 77-85, 2000. doi:10.1115/1.429629

[34] Santamarina, A., Weydahl, E., Siegel, J.M. Jr., \& Moore, J.E. Jr., Computational analysis of flow in a curved tube model of the coronary arteries: effects of time-varying curvature. Annals of Biomedical Engineering, 26, pp. 944-954, 1998. doi:10.1114/1.113

[35] Delfino, A., Stergiopulos, N., Moore, J.E. Jr., \& Meister, J.J., Residual strain effects on the stress field in a thick-wall finite-element model of the human carotid bifurcation. Journal of Biomechanics, 30, pp. 777-786, 1997. doi:10.1016/S0021-9290(97)00025-0

[36] Fry, D.L., Acute vascular endothelial changes associated with increased blood velocity gradients. Circulation Research, 22, pp. 165-197, 1968.

[37] Nerem, R.M., Vascular fluid mechanics, the arterial wall, and atherosclerosis. Journal of Biomechanical. Engineering, 114, pp. 274-282, 1992. 